\title{
Control of a novel chaotic fractional order system using a state feedback technique
}

\author{
Abolhassan Razminia ${ }^{a}$, Delfim F.M. Torres ${ }^{\mathrm{b}, *}$ \\ a Department of Electrical Engineering, School of Engineering, Persian Gulf University, Bushehr, Iran \\ ${ }^{\mathrm{b}}$ CIDMA-Center for Research and Development in Mathematics and Applications, Department of Mathematics, University of Aveiro, $3810-193$ Aveiro, Portugal
}

\section{A R T I C L E I N F O}

\section{Article history:}

Received 6 December 2012

Revised 2 March 2013

Accepted 5 March 2013

Available online 10 April 2013

\section{Keywords:}

Chaos

Control of chaos

Fractional order systems

Stability tests

\begin{abstract}
A B S T R A C T
We consider a new fractional order chaotic system displaying an interesting behavior. A necessary condition for the system to remain chaotic is derived. It is found that chaos exists in the system with order less than three. Using the Routh-Hurwitz and the Matignon stability criteria, we analyze the novel chaotic fractional order system and propose a control methodology that is better than the nonlinear counterparts available in the literature, in the sense of simplicity of implementation and analysis. A scalar control input that excites only one of the states is proposed, and sufficient conditions for the controller gain to stabilize the unstable equilibrium points derived. Numerical simulations confirm the theoretical analysis.
\end{abstract}

(c) 2013 Elsevier Ltd. All rights reserved.

\section{Introduction}

Fractional calculus has been known since the early 17th century [1]. It has been extensively applied in many fields, with an overwhelming growth of applications during the last three decades. Examples abound in physics [2], engineering [3], mathematical biology [4], finance [5], life sciences [6], and optimal control [7]. This is due to the fact that, in many applications, approaches based on fractional derivatives establish far superior models of engineering systems than the approaches based on ordinary derivatives [8]. As mentioned in [1], there is no field that has remained untouched by fractional derivatives.

Historically, the lack of a physical interpretation of fractional derivatives has been acknowledged at the first international conference on the fractional calculus in New Haven (USA), in 1974, by including it in the list of open problems [9]. The question was not answered, and therefore repeated at the subsequent conferences at the University of Strathclyde (UK) in 1984 [10] and at the Nihon University (Tokyo, Japan) in 1989 [11]. The round-table discussion at the conference on transform methods and special functions in Varna, in 1996, showed that the problem was still unsolved [12]. Since then, the geometric and physical interpretation of fractional derivatives has been studied by several authors $[13,14]$. An interesting physical discussion about initial conditions of fractional order systems is reported in [15], and their role in control theory is addressed in [16] for Caputo fractional derivatives,

\footnotetext{
* Corresponding author. Tel.: +351234370668.

E-mail addresses: a.razminia@gmail.com (A. Razminia), delfim@ua.pt (D.F.M. Torres).
}

and in [17] for Riemann-Liouville fractional derivatives. Relation between fractional integrals and derivatives and fractal geometry, showing that for some complex systems substitution of integer-order derivatives by fractional ones results in more accurate and superior models, has received special attention $[18,19]$.

Chaos is an interesting phenomenon in nonlinear dynamical systems that has been developed and thoroughly studied over the past two decades. The reader interested in applications of chaos in medicine and biology, where fractional calculus has initiated its success and activity in engineering applications, is referred to $[20,21]$. A chaotic system is a nonlinear deterministic system that displays complex, noisy-like and unpredictable behavior. The sensitive dependence on the initial conditions, and the system parameter variation, is a prominent characteristic of chaotic behavior. Here we consider a fractional order chaotic system. The corresponding integer-order system has a chaotic behavior for a wide range of parameters. Such integer-order dynamical system is a reduced model for a physical system. When such system is implemented using physical electronic devices, the environment effects (aging of the elements, temperature, inaccurate values, and so on) on the elements appear as a different behavior so that the response predicted by the model does not resemble the actual system. Indeed, for such chaotic systems the super sensitivity of the values of the elements to tiny changes, cannot be considered in the dynamical equations. The main reason is related to the fractal or holed basin of the invariant set of the system. In other words, some trace of the system trajectories are seen in the observed coordinate (phase plane) and some of them lie in the unseen region that cannot be handled by the classical nonlinear ODE model. For this reason, the fractional order system is more appropriate than 
the classical one $[9-13,18,19]$. This happens to be a frequent situation: for most physical systems which exhibit chaotic behavior, the invariant set is not an integer-order dimensional object, and the basin of the trajectories in the phase space is a strange attractor field whose Lyapunov dimensions are non-integer. The fractional order operators allow to describe the observed behavior by an appropriate kernel in the integral. This kernel can be treated as a weighting factor that generates a new response using existing vector fields.

The study of fractional order dynamical systems has attracted an increasing attention in recent years due to their great promise as a valuable tool in the modeling of many phenomena [22]. As a matter of fact, real world processes generally or most likely are fractional order systems [23]. It has been found that fractional order systems possess memory and display more sophisticated dynamics when compared to their integer order counterparts, which is of great significance in secure communications. On the other hand, due to their potential applications in laser physics, chemical reactors, secure communications and economics, a new direction of chaos research has emerged in the past two decades to address the more challenging problem of chaos synchronization and control [24-27]. Recent papers study the control and synchronization of chaotic systems in both integer and fractional order cases, applying various control methodologies: nonlinear control $[28,29]$, adaptive control [30,31], robust control [32], fuzzy control [33], and active control [34]. The main problem in applying such control methodologies for taming chaos is often their complexity in implementation. In this paper we propose a simple linear control mechanism, based on the well-known Routh-Hurwitz stability criterion, for a fractional order dynamical system. The system shows very rich nonlinear dynamics, including chaos and period doubling bifurcations. A controller is proposed, and both analysis and design are studied. We show that a single input can control the very complex system. This makes our results simpler than the conventional nonlinear methods available in the literature and more feasible to implement.

The rest of the manuscript is organized as follows. Section 2 briefly presents the necessary fractional calculus background. In Section 3 we recall the stability criteria that are used in our subsequent analysis and design. Description of an interesting system is presented in Section 4, while the new results are given in Section 5, where we illustrate our control methodology for taming the fractional order chaotic system corresponding to the one of Section 4. Conclusions and future directions of research are given in Section 6.

\section{Fractional calculus background}

In this section some necessary mathematical background is presented. For more details see the books [35-39].

Definition 1. (see, e.g., [40]) The (left) fractional integral of $x$ of order $q, q \in \mathbb{R}^{+}$, is defined by

${ }_{a} D_{t}^{-q} x(t):=\frac{1}{\Gamma(q)} \int_{a}^{t}(t-s)^{q-1} x(s) d s$,

where $\Gamma(q)=\int_{0}^{\infty} e^{-z} z^{q-1} d z$ is the Gamma function.

Definition 2. (see, e.g., [29]) The (left) fractional derivative of $x$ of order $q, q \in \mathbb{R}^{+}$, in the sense of Riemann-Liouville, is defined by

${ }_{a}^{R L} D_{t}^{q} q x(t):=D^{m}{ }_{a} D_{t}^{-(m-q)} x(t)=\frac{1}{\Gamma(m-q)} \frac{d^{m}}{d t^{m}} \int_{a}^{t}(t-s)^{m-q-1} x(s) d s$,

where $m \in \mathbb{Z}^{+}$is such that $m-1<q<m$.
Theorem 3 (see, e.g., [41]). For the fractional Riemann-Liouville derivative and integral one has:

1. $\mathcal{L}\left\{{ }_{a} D_{t}^{-q} x(t)\right\}=s^{-q} X(s)$;

2. $\lim _{q \rightarrow m 0} D_{t}^{-q} x(t)=D^{-m} x(t), q>0, m \in \mathbb{Z}^{+}$;

3. ${ }_{0}^{R L} D_{t}^{q} c=\frac{c t^{q-1}}{\Gamma(1-q)}$;

4. ${ }_{0}^{R L} D_{t 0}^{q} D_{t}^{-q} x(t)=x(t), q \in \mathbb{R}^{+}$;

5. $\mathcal{L}\left\{{ }_{0}^{R L} D_{t}^{q} x(t)\right\}=s^{q} X(s)-\sum_{k=0}^{m-1} s^{k} \cdot{ }_{0}^{R L} D_{t}^{q-k-1} x(0), m-1<q<m, m$ $\in \mathbb{Z}^{+}$;

where $\mathcal{L}$ denotes the Laplace transform, $c$ a constant, and $D^{-m}$ the $m$-folded integral.

We note that the Riemann-Liouville differentiation of a constant is not zero (item 3 of Theorem 3); while its Laplace transform needs fractional derivatives of the function in initial time (item 5 of Theorem 3). To overcome these "imperfections", the Caputo fractional derivative has been introduced.

Definition 4 (see, e.g., [29]). The (left) fractional derivative of $x$ of order $q, q \in \mathbb{R}^{+}$, in the sense of Caputo, is defined by

${ }_{a}^{C} D_{t}^{q} x(t):={ }_{a}^{R L} D_{t}^{-(m-q)} D^{m} x(t)=\frac{1}{\Gamma(m-q)} \int_{a}^{t}(t-s)^{m-q-1} x^{(m)}(s) d s$,

where $m \in \mathbb{Z}^{+}$is such that $m-1<q<m, D^{m}$ is the standard differential operator of order $m$.

Theorem 5 (see, e.g., [30]). For the fractional Caputo derivative one has:

1. ${ }_{0}^{C} D_{t}^{-q} c=0$;

2. ${ }_{0}^{C} D_{t}^{-q} x(t)={ }_{0}^{R L} D_{t}^{-q}{ }_{0} D_{t}^{-q} x(t)=x(t), 0<q<1$;

3. $\mathcal{L}\left\{{ }_{0}^{C} D_{t}^{q} x(t)\right\}=s^{q} X(s)-\sum_{k=0}^{m-1} s^{q-k-1} x^{(k)}(0), m-1<q<m, m \in$ $\mathbb{Z}^{+}$;

where $c$ denotes a constant and $\mathcal{L}$ the Laplace transform.

In the next section we study some stability tests for fractional order systems.

\section{Stability criteria}

A fractional order dynamical system is usually described by

$\begin{cases}{ }_{0}^{C} D_{t}^{q} x(t)=f(x(t), t), & m-1<q<m, m \in \mathbb{Z}^{+}, t>0, \\ {\left.\left[{ }_{0}^{R L} D_{t}^{k} x(t)\right]\right|_{t=0}=x_{0}^{k},} & k=0, \ldots, m-1,\end{cases}$

where $x(t) \in \mathbb{R}^{n}$ is the vector state at time $t, f: \mathbb{R}^{n} \times \mathbb{R} \rightarrow \mathbb{R}^{n}$ the nonlinear vector field, and $q=\left(q_{1}, \ldots, q_{n}\right)^{T}$ the differentiation order vector. If $q_{1}=q_{2}=\cdots=q_{n}=: \alpha$, we call (1) a commensurate fractional order dynamical system; otherwise, (1) is said to be incommensurate. The sum of the orders of all involved derivatives in Eq. (1), i.e., $\sum_{i=1}^{n} q_{i}$, is called the effective dimension of Eq. (1) [42]. The size of vector $x(t)$ in state space form (1), i.e., $n$, is called the inner dimension of system (1) [43].

Theorem 6 (see [33]). The commensurate order system

${ }_{0}^{C} D_{t}^{\alpha} x(t)=A x(t), \quad x(0)=x_{0}$,

with $0<\alpha \leqslant 1, x(t) \in \mathbb{R}^{n}$, and $A \in \mathbb{R}^{n \times n}$, is asymptotically stable if, and only if, $|\arg (\lambda)|>\alpha \frac{\pi}{2}$ for all eigenvalues $\lambda$ of A. Moreover, system (2) is stable if, and only if, $|\arg (\lambda)| \geqslant \alpha \frac{\pi}{2}$ for all eigenvalues $\lambda$ of $A$, with those critical eigenvalues satisfying $|\arg (\lambda)|=\alpha \frac{\pi}{2}$ having geometric multiplicity of one. 
Theorem 7 (see [44]). Consider the following linear fractional order system:

${ }_{0}^{C} D_{t}^{q} x(t)=A x(t), \quad x(0)=x_{0}$,

where $x(t) \in \mathbb{R}^{n}, A \in \mathbb{R}^{n \times n}$, and $q=\left(q_{1}, \ldots, q_{n}\right)^{T}, 0<q_{i} \leqslant 1, q_{i}=\frac{n_{i}}{d_{i}}$ and gcd $\left(n_{i}, d_{i}\right)=1, i=1, \ldots, n$. If $M$ is the least common multiple of the denominators $d_{i}, i=1, \ldots, n$, then the zero solution of (3) is globally asymptotically stable in the Lyapunov sense if all roots $\lambda$ of equation

$\Delta(\lambda)=\operatorname{det}\left(\operatorname{diag}\left(\lambda^{M q_{i}}\right)-A\right)=0$

satisfy $|\arg (\lambda)|>\frac{\pi}{2 M}$.

Theorem 8 (see [45]). Let $Q=\left(x^{*}, y^{*}, z^{*}\right)$ be an equilibrium solution of (1) when $n=3$ and $0<q_{1}=q_{2}=q_{3}=: \alpha \leqslant 1$; and the eigenvalues of the equilibrium point $Q$ for the Jacobian matrix $J:=\frac{\partial f}{\partial x} \mid$ be given by the polynomial $\Delta(\lambda)=\lambda^{3}+a_{1} \lambda^{2}+a_{2} \lambda+a_{3}=0$ with discriminant

$D(\Delta)=18 a_{1} a_{2} a_{3}+\left(a_{1} a_{2}\right)^{2}-4 a_{3}\left(a_{1}\right)^{3}-4\left(a_{2}\right)^{3}-27\left(a_{3}\right)^{2}$.

The following holds:

(i) If $D(\Delta)>0$, then a necessary and sufficient condition for the equilibrium point $Q$ to be locally asymptotically stable is $a_{1}>0, a_{3}>0, a_{1} a_{2}-a_{3}>0$.

(ii) If $D(\Delta)<0$ and $a_{1} \geqslant 0, a_{2} \geqslant 0, a_{3} \geqslant 0$, then $Q$ is locally asymptotically stable for $\alpha<\frac{2}{3}$. However, if $D(\Delta)<0, a_{1}<0, a_{2}<0, \alpha>\frac{2}{3}$, then all roots of Eq. (5) satisfy the condition $|\arg (\lambda)|<\alpha \frac{\pi}{2}$.

(iii) If $D(\Delta)<0, a_{1}>0, a_{2}>0, a_{1} a_{2}-a_{3}=0$, then $Q$ is locally asymptotically stable for all $\alpha \in(0,1)$.

(iv) A necessary condition for the equilibrium point $Q$ to be locally asymptotically stable is $a_{3}>0$.

(v) If the conditions $D(\Delta)<0, a_{1}>0, a_{2}>0, a_{1} a_{2}-a_{3}=0$ are satisfied, then the equilibrium point $Q$ is not locally asymptotically stable for $\alpha=1$.

\section{An interesting system}

In [46] the following three-dimensional smooth system is proposed and investigated:

$$
\left(\begin{array}{c}
\dot{x} \\
\dot{y} \\
\dot{z}
\end{array}\right)=\left(\begin{array}{c}
y-a x+b y z \\
c y-x z+z \\
d x y-h z
\end{array}\right),
$$

where $[x(t), y(t), z(t)]^{T} \in \mathbb{R}^{3}$ is the state vector, and $a, b, c, d$, and $h$ are some positive constants. The system was shown to be chaotic in a wide parameter range, and to have an interesting complex dynamical behavior that varies according with the values of the parameters $a, b$, $c, d$, and $h$. The very rich nonlinear dynamics include chaos and period doubling bifurcations. In particular, the system generates a twoscroll chaotic attractor for $(a, b, c, d, h)=(3,2.7,4.7,2,9)$.

Chaos may be seen in many real-life nonlinear systems. About 10 years ago, several experimental and theoretical studies have been done to depict the chaotic behavior in various electronic systems: nonlinear circuits [47], secure communications [48,49], lasers [50], and Colpitts oscillators [51]. The system (6) is particularly relevant in mechatronics, where it can be used as a carrier producer. Indeed, because of its wide range chaoticity, one of its important applications is in secure communication systems. In such a system, a chaotic carrier is used to transmit the message signal over a channel. The main motivation for employing such carriers is: (i) complexity of the carrier, which increases the security of the modulated signal; (ii) inherent orthonormality of the chaotic signals, avoiding the necessity to use in-phase local oscillators as often done in tele- communication systems in order to demodulate the original signal at the receiver; (iii) wide band signal, which permits the carrier to transmit a wide band message over a noisy channel. Motivated by the interesting behavior of (6) obtained in [46], our main goal is to investigate the chaotic dynamics of the corresponding fractional system. This is done in the next section.

\section{Main results}

Consider a 3D autonomous fractional system

${ }_{0}^{C} D_{t}^{q} x(t)=f(x(t))$,

where $q=\left(q_{1}, q_{2}, q_{3}\right)^{T}$ is the fractional order of differentiation, $x(t) \in \mathbb{R}^{3}$ is the state vector, and $f: \mathbb{R}^{3} \rightarrow \mathbb{R}^{3}$ is the nonlinear vector field. Let $Q=\left(x_{1}^{*}, x_{2}^{*}, x_{3}^{*}\right)$ be an equilibrium of the system (7), i.e., let $f(Q)=0$. We say that $Q$ is a saddle point for (7) if the eigenvalues of the Jacobian matrix $J=\frac{\partial f}{\partial x}$ evaluated at $Q$ are $a$ and $b \pm j c$, where $a b<0$ and $c \neq 0$. A saddle point $Q$ is called a saddle point of index 1 if $a>0$ and $b<0$, and it is called a saddle point of index 2 if $a<0$ and $b>0$. In chaotic systems of Shil'nikov type, scrolls in a chaotic attractor are generated only around the saddle points of index 2 . Moreover, saddle points of index 1 are responsible only for connecting scrolls [52].

\subsection{System description}

We are interested in the particular case of (7) that corresponds to the commensurate fractional order version of (6). For that we substitute the standard/integer order derivatives in (6) by Caputo fractional derivatives of order $\alpha \in(0,1)$ :

$\left(\begin{array}{l}{ }_{0}^{c} D_{t}^{\alpha} x \\ { }_{0}^{c} D_{t}^{\alpha} y \\ { }_{0}^{c} D_{t}^{\alpha} z\end{array}\right)=\left(\begin{array}{c}y-a x+b y z \\ c y-x z+z \\ d x y-h z\end{array}\right)$.

System (8) can be used to model several mechatronic systems. One possible application is to model the nonlinear dynamics of a rotor-bearing system with the purpose of diagnosing malfunctions and effectively improve the dynamic characteristics of the rotor system. Chu and Zhang analyzed the bifurcation and chaotic motion of a rub-impact rotor system. They found three different routes to chaos with an increasing rotating speed [53]. Later, Chu observed very rich forms of periodic and chaotic vibrations through experimental verification. These results are of great importance to the fault diagnosis of the rub-impact problem [54]. Ehrich studies the bifurcation of a bearing-rotor system, identifying a sub-harmonic vibration phenomenon in a rotor dynamic system [55]. Goldman and Muszynska analyze the chaotic behavior of a rub-impact rotor using numerical emulation and simple experimental verification. They conclude that the rub can lead to higher order harmonics, sub-harmonic fractional frequencies, or to chaotic vibrations [56]. Lin et al. analyze the nonlinear behavior of rub-related vibration in rotating machinery: the effects of the rotating speed, clearance, damping factors, friction coefficients, and boundary stiffness are investigated [57]. Our system (8) can also be regarded as a model for a DC-motor with chaotic behavior (self-sustained oscillations according to backlash and dead-zone of the gears) [58]. A schematic diagram of a DC drive and its circuits, with separate excitation and controller with hysteresis, can be found in [58, Fig. 1]. The states of such system are the current $i_{a}$ in the motor armature circuit; the current $i_{f}$ in the excitation coil; and the rotor angular speed $\omega_{r}$. This real life system can be described by (8), after normalization and rescale according to the parameters and control signals of the circuit, with the correspondence $x \leftrightarrow \omega_{r}, y \leftrightarrow i_{f}$, and $z \leftrightarrow i_{a}$. 
To find the equilibria of system (8), it is enough to equate the right-hand side of (8) to zero: $y-a x+b y z=0, c y-x z+z=0$, $d x y-h z=0$. One concludes that the system has 5 equilibria:

$Q_{1}=(0,0,0), Q_{2}=\left(\frac{d+\sqrt{\Delta}}{2 d}, \frac{h}{b}\left(\frac{-1+\sqrt{1+\Lambda}}{d+\sqrt{\Delta}}\right), \frac{-1+\sqrt{1+\Lambda}}{2 b}\right)$,

$Q_{3}=\left(\frac{d+\sqrt{\Delta}}{2 d}, \frac{h}{b}\left(\frac{-1-\sqrt{1+\Lambda}}{d+\sqrt{\Delta}}\right), \frac{-1-\sqrt{1+\Lambda}}{2 b}\right)$,

$Q_{4}=\left(\frac{d-\sqrt{\Delta}}{2 d}, \frac{h}{b}\left(\frac{-1+\sqrt{1+\Lambda}}{d-\sqrt{\Delta}}\right), \frac{-1+\sqrt{1+\Gamma}}{2 b}\right)$,

$Q_{5}=\left(\frac{d-\sqrt{\Delta}}{2 d}, \frac{h}{b}\left(\frac{-1-\sqrt{1+\Lambda}}{d-\sqrt{\Delta}}\right), \frac{-1-\sqrt{1+\Gamma}}{2 b}\right)$,

where $\Delta=d^{2}+4 c h d, \Lambda=\frac{2 a b}{h}(d+2 c h+\sqrt{\Delta})$, and $\Gamma=\frac{2 a b}{h}(d+2 c h-$ $\sqrt{\Delta}$ ). The Jacobian matrix for (8), evaluated in an equilibrium point $Q=\left(x^{*}, y^{*}, z^{*}\right)$, is given by

$J=\left(\begin{array}{ccc}-a & 1+b z^{*} & b y^{*} \\ -z^{*} & c & 1-x^{*} \\ d y^{*} & d x^{*} & -h\end{array}\right)$.

Our first result gives a necessary condition on the fractional order of differentiation $\alpha$, for chaos to occur.

Theorem 9. [Necessary condition for occurrence of a chaotic attractor in the fractional order system (8)] If the fractional order system (8) exhibits a chaotic attractor, then

$\alpha>\frac{2}{\pi} \arctan \left(\frac{|\operatorname{Im}(\lambda)|}{\operatorname{Re}(\lambda)}\right)$

for any eigenvalue $\lambda$ of $Q_{i}$ in (9), $i=1, \ldots, 5$.

Proof. Assume that the 3D fractional system (8) displays a chaotic attractor. For every scroll existing in the chaotic attractor, the system has a saddle point of index 2 encircled by its respective scroll. Suppose that $\Omega$ is the set of equilibrium points of the system surrounded by scrolls. A necessary condition for the fractional order system (8) to exhibit a chaotic attractor is instability of the equilibrium points in $\Omega$ [59]. Otherwise, one of these equilibrium points becomes asymptotically stable and attracts the nearby trajectories. According to (4), this necessary condition is mathematically equivalent to
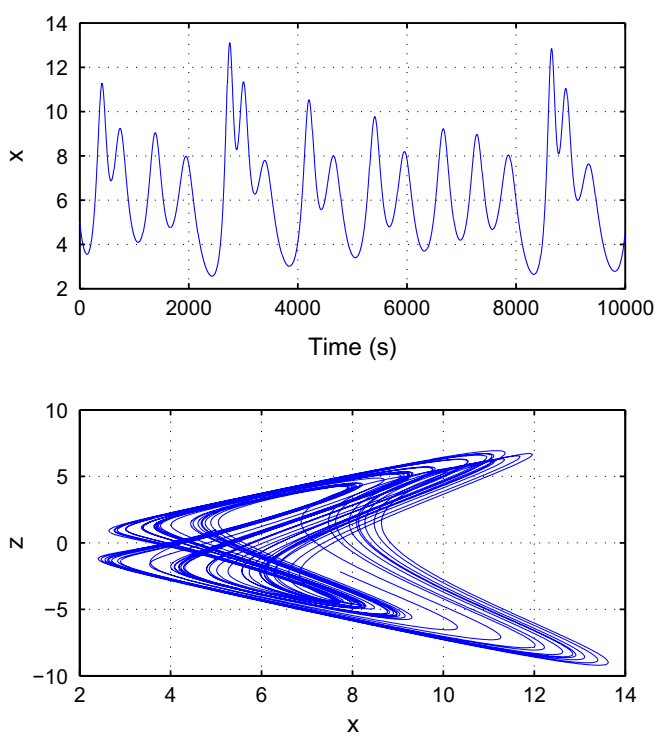

$\frac{\pi}{2 M}-\min _{i}\left\{\left|\arg \left(\lambda_{i}\right)\right|\right\} \geqslant 0$,

where the $\lambda_{i}$ are the roots of $\operatorname{det}\left(\operatorname{diag}\left(\lambda^{M q_{1}} \lambda^{M q_{2}} \lambda^{M q_{3}}\right)-\left.J\right|_{Q}\right)=0$ for all $Q \in \Omega$. Condition (10) follows immediately from (11).

The nature of the equilibria (9) of (8) may be determined using the corresponding eigenvalues $\lambda$. The following proposition lists the eigenvalues of each equilibrium, when the parameters are selected in agreement with Section 4.

Proposition 10. Consider the fractional system (8) of commensurate order $\alpha \in(0,1)$, when the five parameters are selected to be ( $a, b, c, d$, $h)=(3,2.7,4.7,2,9)$. Then, the eigenvalues $\lambda$ for each equilibrium $Q_{i}$ in (9), $i=1, \ldots, 5$, are given as follows.

(i) Eigenvalues of $Q_{1}:-9,-3$ and 4.7 .

(ii) Eigenvalues of $Q_{2}:-11.0247$ and $1.8623 \pm j 6.6831$.

(iii) Eigenvalues of $Q_{3}$ : -11.7856 and $2.2428 \pm j 6.8580$.

(iv) Eigenvalues of $Q_{4}:-10.7669$ and $1.7335 \pm j 6.0024$.

(v) Eigenvalues of $Q_{5}:-11.6813$ and $2.1906 \pm j 6.1881$.

Proof. Follows by direct computations.

Corollary 11. For the fractional system (8) with ( $a, b, c, d$, $h)=(3,2.7,4.7,2,9)$, the equilibria $Q_{2}, Q_{3}, Q_{4}$ and $Q_{5}$ are saddle points of index 2.

We conclude that if there are some chaotic attractors for $(a, b, c$, $d, h)=(3,2.7,4.7,2,9)$, they are located around the equilibria $Q_{2}, Q_{3}$, $Q_{4}, Q_{5}$. Examining (10) for these equilibria, we obtain: $\alpha>0.8270$ for $Q_{2} ; \alpha>0.7988$ for $Q_{3} ; \alpha>0.8210$ for $Q_{4} ;$ and $\alpha>0.7834$ for $Q_{5}$. Therefore, by choosing $\alpha>0.8270$, we ensure that all the eigenvalues remain in the instability region.

An efficient method for solving fractional order differential equations is the predictor-corrector scheme or, more precisely, the PECE (Predict, Evaluate, Correct, Evaluate) technique that has been investigated in $[60,61]$. It represents a generalization of the Adams-Bashforth-Moulton algorithm. We use the PECE scheme throughout the paper for numerical simulations.

In Figs. 1-5, the initial conditions were selected as $\left(x_{0}, y_{0}\right.$, $\left.z_{0}\right)=(5,-2,1)$, and only the fractional order of differentiation $\alpha$ changes. When $\alpha \rightarrow 1$, our numerical results are in agreement with
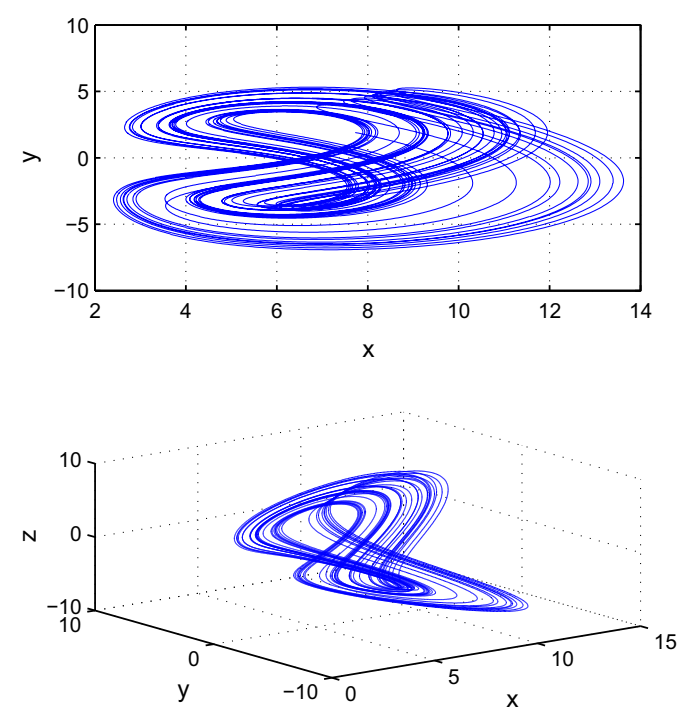

Fig. 1. Chaotic attractor of fractional system (8) with $a=3, b=2.7, c=4.7, d=2, h=9$, and initial conditions $\left(x_{0}, y_{0}, z_{0}\right)=(5,-2,1)$, when $\alpha \rightarrow 1$. 

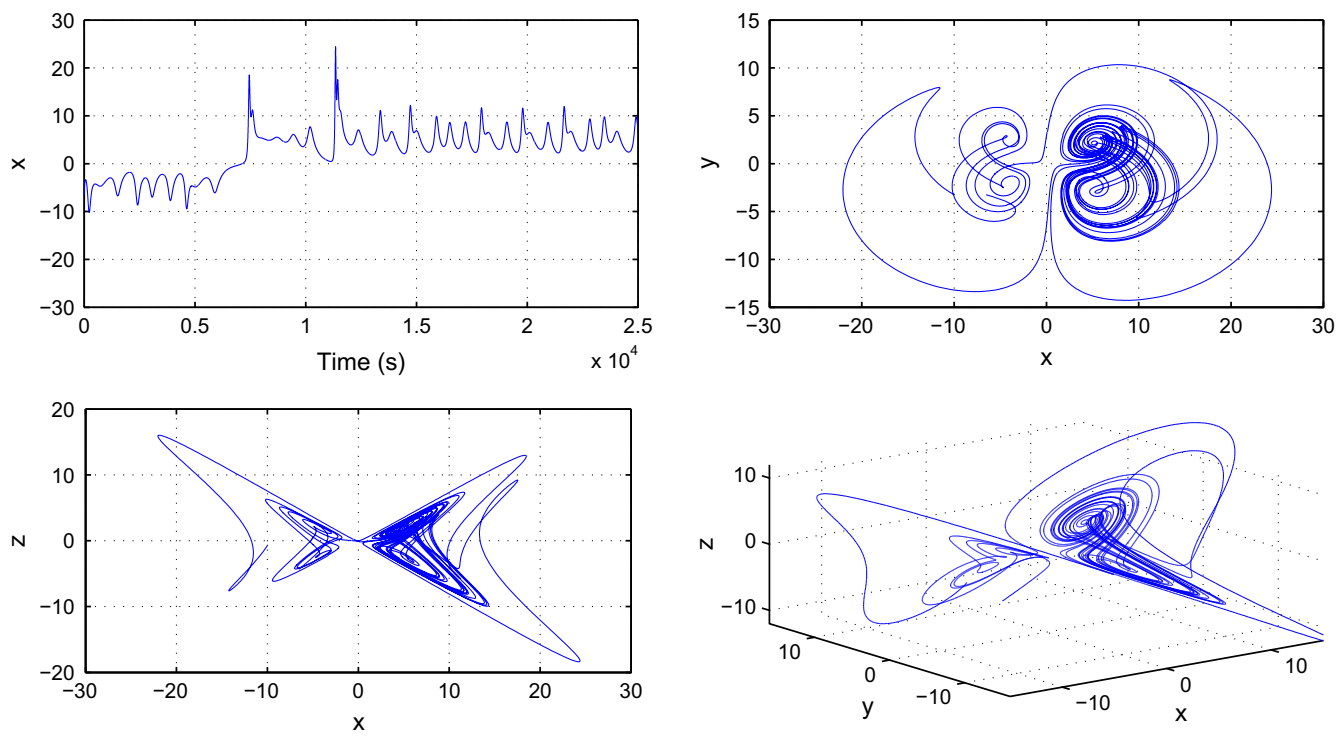

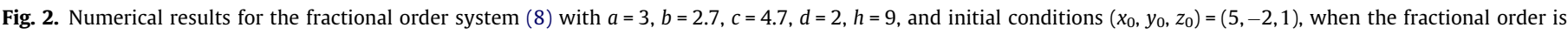
$\alpha=0.90$.
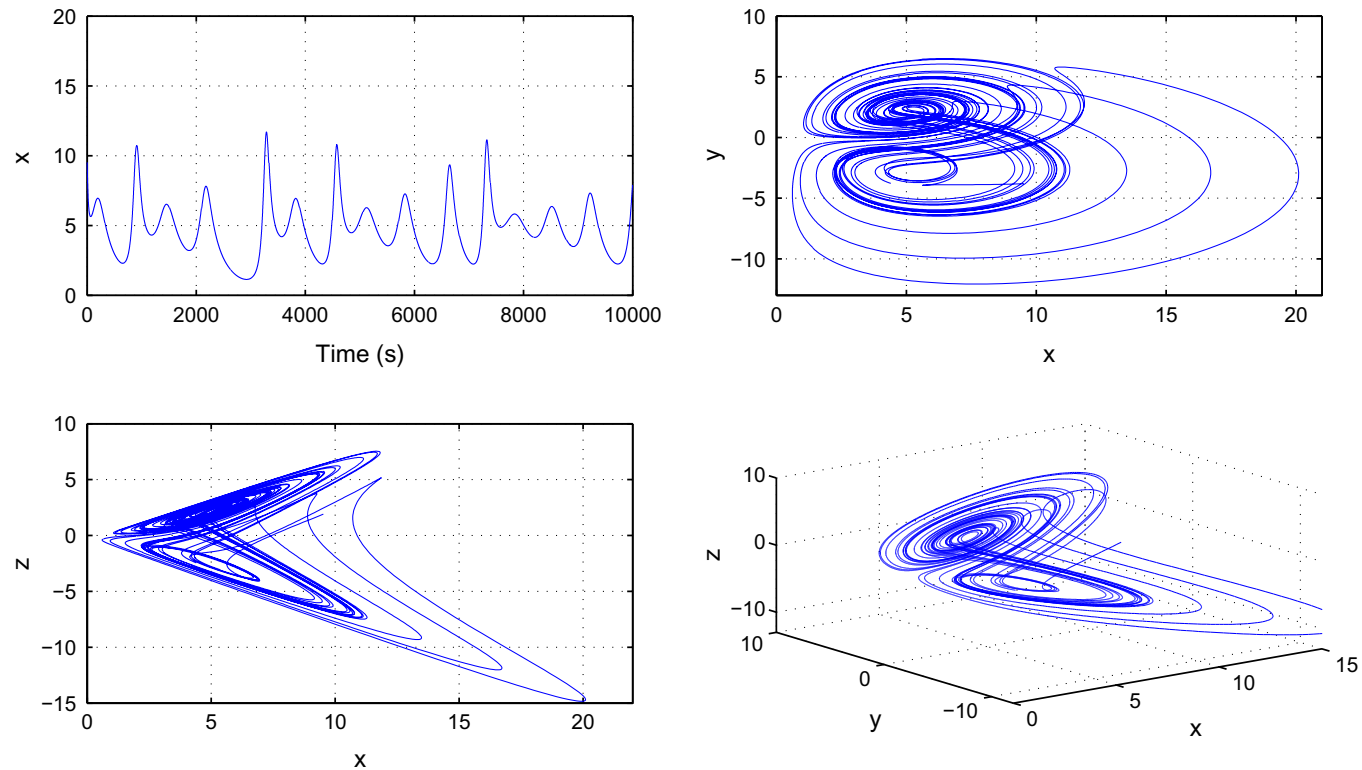

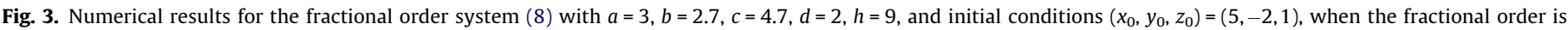
$\alpha=0.86$.

[46]. The numerical simulation of the chaotic attractor for $\alpha \rightarrow 1$ is depicted in Fig. 1.

In Fig. 2 we illustrate the chaotic behavior of (8) when $\alpha=0.90$, and in Fig. 3, Fig. 4, and Fig. 5, the behavior of (8) is depicted for $\alpha=0.86, \alpha=0.80$, and $\alpha=0.77$, respectively.

As one can see, in Figs. 4 and 5 chaos has diminished, and the trajectories of the corresponding fractional system converge to their equilibria.

\subsection{Control of the fractional order chaotic system}

Consider the fractional order chaotic system (8) of commensurate order $\alpha \in(0,1)$. In order to control the system, i.e., force the trajectories to go to the equilibria, we add a control parameter $u=\left(u_{1}, u_{2}, u_{3}\right)$ as follows: $\left(\begin{array}{l}{ }_{0}^{c} D_{t}^{\alpha} x \\ { }_{0}^{c} D_{t}^{\alpha} y \\ { }_{0}^{c} D_{t}^{\alpha} z\end{array}\right)=\left(\begin{array}{c}y-a x+b y z \\ c y-x z+z \\ d x y-h z\end{array}\right)+\left(\begin{array}{l}u_{1} \\ u_{2} \\ u_{3}\end{array}\right)$.

One of the simplest controllers is the state feedback controller, which has a simple structure and is easy to implement. Let us take the structure of the controller as a state feedback, as follows:

$\left\{\begin{array}{l}u_{1}=-k_{1}\left(x-x^{*}\right) \\ u_{2}=-k_{2}\left(y-y^{*}\right) \\ u_{3}=-k_{3}\left(z-z^{*}\right)\end{array}\right.$

In this way (12) reduces to 

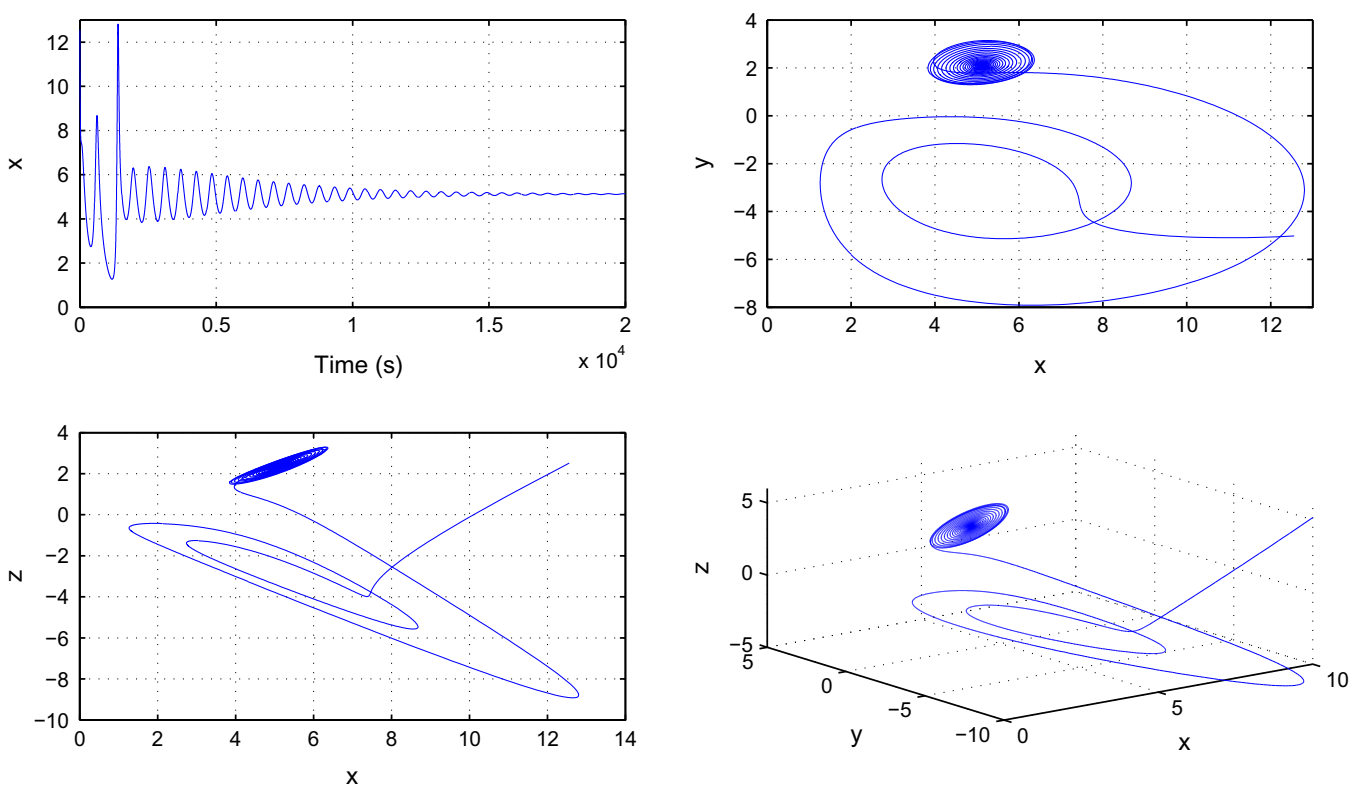

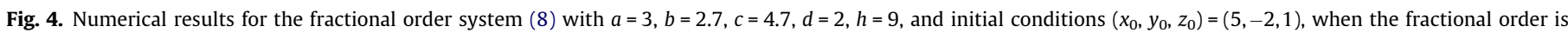
$\alpha=0.80$.
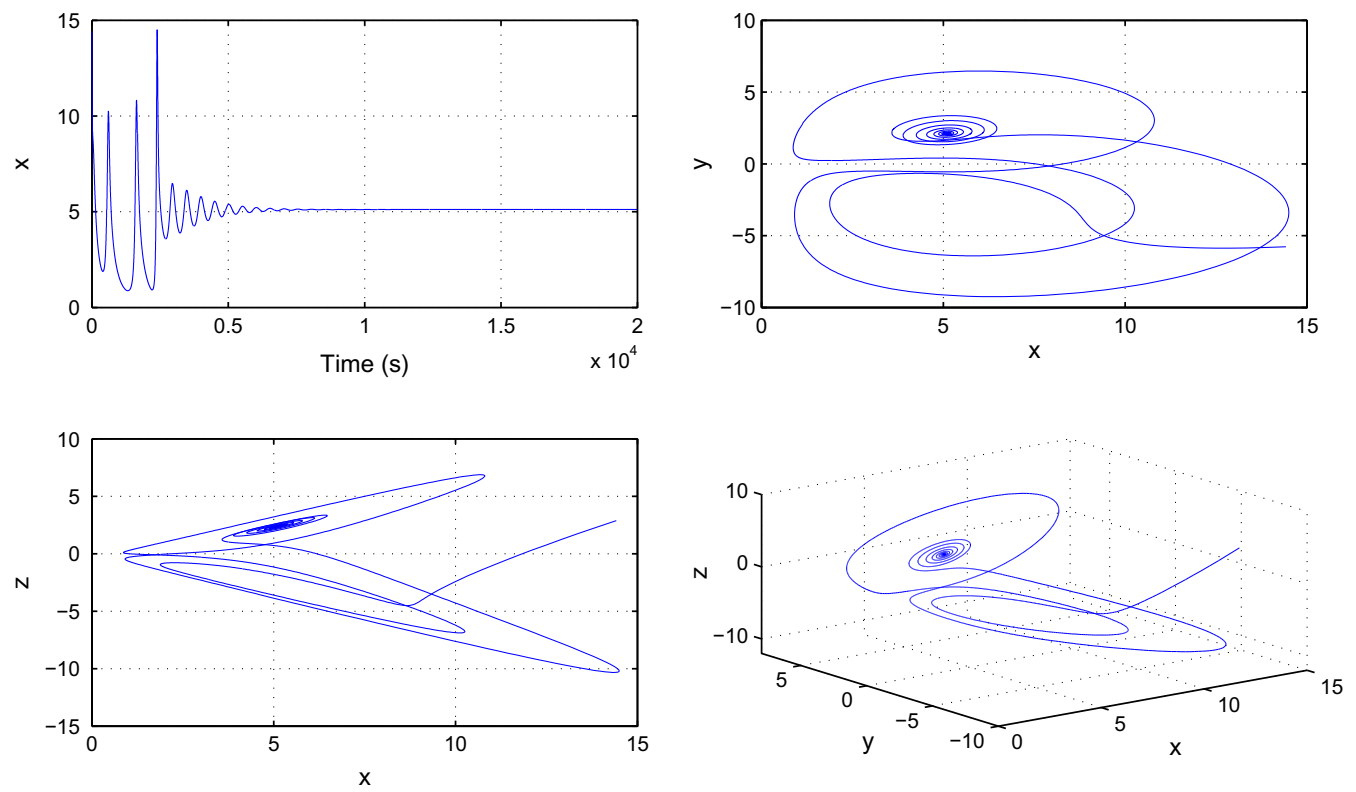

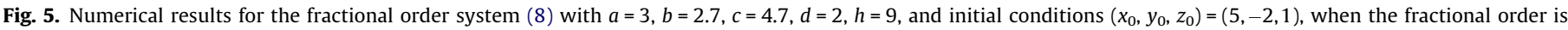
$\alpha=0.77$.

$$
\left(\begin{array}{l}
{ }_{0}^{C} D_{t}^{\alpha} x \\
{ }_{0}^{C} D_{t}^{\alpha} y \\
{ }_{0}^{c} D_{t}^{\alpha} z
\end{array}\right)=\left(\begin{array}{c}
y-a x+b y z-k_{1}\left(x-x^{*}\right) \\
c y-x z+z-k_{2}\left(y-y^{*}\right) \\
d x y-h z-k_{3}\left(z-z^{*}\right)
\end{array}\right) \text {. }
$$

Assume that we want to stabilize one of the equilibria, e.g., $Q_{2}$ (using a similar approach, we can easily design a control law to stabilize the other unstable equilibria). Next theorem shows that by choosing appropriate values for gain $k_{1}$, we can control the fractional order system (13).

Theorem 12. The trajectories of the fractional controlled system (13) with $a=3, b=2.7, c=4.7, d=2$, and $h=9$, are driven to the unstable equilibrium point $Q_{2}=(5.1260,2.0794,2.3687)$ for all $\alpha \in(0,1)$, if $k_{2}=k_{3}=0$ and $-7.30<k_{1}<26.53$.
Proof. Computing the Jacobian matrix in the equilibrium point $Q=\left(x^{*}, y^{*}, z^{*}\right)$, we obtain

$J=\left(\begin{array}{ccc}-a-k_{1} & 1+b z^{*} & b y^{*} \\ -z^{*} & c-k_{2} & -x^{*}+1 \\ d y^{*} & d x^{*} & -h-k_{3}\end{array}\right)$.

Constituting the characteristic equation $\Delta(\lambda)$ by

$$
\Delta(\lambda)=\operatorname{det}(\lambda I-J)=\operatorname{det}\left(\begin{array}{ccc}
\lambda+a+k_{1} & -1-b z^{*} & -b y^{*} \\
z^{*} & \lambda-c+k_{2} & x^{*}-1 \\
-d y^{*} & -d x^{*} & \lambda+h+k_{3}
\end{array}\right)=0,
$$

we have 


$$
\begin{aligned}
\Delta(\lambda)= & \left(\lambda+a+k_{1}\right)\left[\left(\lambda-c+k_{2}\right)\left(\lambda+h+k_{3}\right)+d x^{*}\left(x^{*}-1\right)\right] \\
& +\left(1+b z^{*}\right)\left[z^{*}\left(\lambda+h+k_{3}\right)+d y^{*}\left(x^{*}-1\right)\right] \\
& -b y^{*}\left[\left(-d x^{*} z^{*}\right)+d y^{*}\left(\lambda-c+k_{2}\right)\right] \\
= & \lambda^{3}+\left(a+k_{1}+k_{2}-c+h+k_{3}\right) \lambda^{2}+\left(d x^{*}\left(x^{*}-1\right)+(a\right. \\
& \left.+k_{1}\right)\left(k_{2}-c+h+k_{3}\right)+\left(k_{2}-c\right)\left(h+k_{3}\right)+\left(1+b z^{*}\right) z^{*} \\
& \left.-b d y^{* 2}\right) \lambda+\left(a+k_{1}\right)\left(d x^{*}\left(x^{*}-1\right)+\left(k_{2}-c\right)\left(h+k_{3}\right)\right) \\
& +\left(1+b z^{*}\right)\left(d y^{*}\left(x^{*}-1\right)+z^{*}\left(h+k_{3}\right)\right)-b y^{*}\left(-d x^{*} z^{*}\right. \\
& \left.+d y^{*}\left(-c+k_{2}\right)\right) \\
= & 0 .
\end{aligned}
$$

Based on Theorem 8, if we choose $k_{1}, k_{2}$ and $k_{3}$ such that $D(\Delta)<0$, $a_{1}>0, a_{2}>0$ and $a_{1} a_{2}-a_{3}=0$, then $Q=\left(x^{*}, y^{*}, z^{*}\right)$ is locally asymptotically stable for all $\alpha \in(0,1)$, where

$$
\begin{aligned}
D(\Delta)= & 18\left(a+k_{1}+k_{2}-c+h+k_{3}\right) \times\left(d x^{*}\left(x^{*}-1\right)+\left(a+k_{1}\right)\left(k_{2}\right.\right. \\
& \left.\left.-c+h+k_{3}\right)+\left(k_{2}-c\right)\left(h+k_{3}\right)+\left(1+b z^{*}\right) z^{*}-b d y^{* 2}\right) \times((a \\
& \left.+k_{1}\right)\left(d x^{*}\left(x^{*}-1\right)+\left(k_{2}-c\right)\left(h+k_{3}\right)\right)+(1 \\
& \left.+b z^{*}\right)\left(d y^{*}\left(x^{*}-1\right)+z^{*}\left(h+k_{3}\right)\right)-b y^{*}\left(-d x^{*} z^{*}+d y^{*}(-c\right. \\
& \left.\left.\left.+k_{2}\right)\right)\right)+\left(( a + k _ { 1 } + k _ { 2 } - c + h + k _ { 3 } ) \left(d x^{*}\left(x^{*}-1\right)+\left(a+k_{1}\right)\right.\right. \\
& \times\left(k_{2}-c+h+k_{3}\right)+\left(k_{2}-c\right)\left(h+k_{3}\right)+\left(1+b z^{*}\right) z^{*} \\
& \left.\left.-b d y^{* 2}\right)\right)^{2}-4\left(\left(a+k_{1}\right)\left(d x^{*}\left(x^{*}-1\right)+\left(k_{2}-c\right)\left(h+k_{3}\right)\right)+(1\right. \\
& \left.+b z^{*}\right)\left(d y^{*}\left(x^{*}-1\right)+z^{*}\left(h+k_{3}\right)\right)-b y^{*}\left(-d x^{*} z^{*}+d y^{*}(-c\right. \\
& \left.\left.\left.+k_{2}\right)\right)\right)\left(a+k_{1}+k_{2}-c+h+k_{3}\right)^{3}-4\left(d x^{*}\left(x^{*}-1\right)+\left(a+k_{1}\right)\right. \\
& \times\left(k_{2}-c+h+k_{3}\right)+\left(k_{2}-c\right)\left(h+k_{3}\right)+\left(1+b z^{*}\right) z^{*} \\
& \left.-b d y^{* 2}\right)^{3}-27\left(\left(a+k_{1}\right)\left(d x^{*}\left(x^{*}-1\right)+\left(k_{2}-c\right)\left(h+k_{3}\right)\right)+(1\right. \\
& \left.+b z^{*}\right)\left(d y^{*}\left(x^{*}-1\right)+z^{*}\left(h+k_{3}\right)\right)-b y^{*}\left(-d x^{*} z^{*}+d y^{*}(-c\right. \\
& \left.\left.\left.+k_{2}\right)\right)\right)^{3}
\end{aligned}
$$

and the $a_{i}, i=1,2,3$, are found from the coefficients of (14). For the parameters that generate the 2-scroll attractors, i.e., $a=3, b=2.7$, $c=4.7, d=2, h=9$, we have $Q_{2}=\left(x^{*}, y^{*}, z^{*}\right)=(5.1260,2.0794$, 2.3687). Thus, (14) reduces to
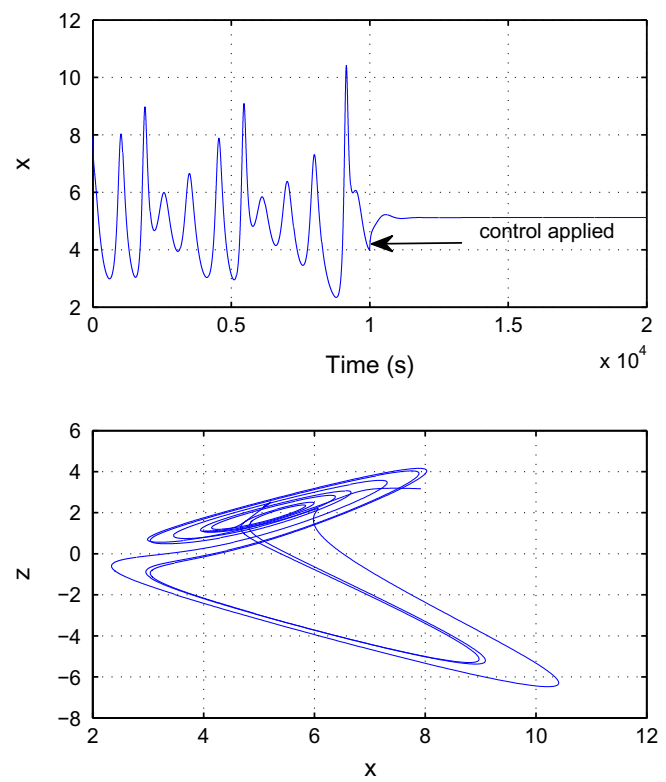

$$
\begin{aligned}
\Delta(\lambda)= & \lambda^{3}+\left(7.3+k_{1}+k_{2}+k_{3}\right) \lambda^{2}+\left(k_{1} k_{2}+k_{2} k_{3}+k_{1} k_{3}\right. \\
& +21.8733) \lambda+530.6404-0.0002 k_{1}+3.6509 k_{2} \\
& +3.4177 k_{3}+3 k_{2} k_{3}+9 k_{1} k_{2}-4.7 k_{1} k_{3}+k_{1} k_{2} k_{3} .
\end{aligned}
$$

If we choose $k_{2}=k_{3}=0$, then

$$
\Delta(\lambda)=\lambda^{3}+\left(7.3+k_{1}\right) \lambda^{2}+21.8733 \lambda+530.6404-0.0002 k_{1} .
$$

For this characteristic polynomial we have:

$$
\begin{aligned}
D(\Delta)= & 18\left(7.3+k_{1}\right)(21.8733)\left(530.6404-0.0002 k_{1}\right)+((7.3 \\
& \left.\left.+k_{1}\right) 21.8733\right)^{2}-4\left(530.6404-0.0002 k_{1}\right)\left(7.3+k_{1}\right)^{3} \\
& -4(21.8733)^{3}-27\left(530.6404-0.0002 k_{1}\right)^{2} .
\end{aligned}
$$

The conditions $D(\Delta)<0, a_{1}>0, a_{2}>0$ and $a_{1} a_{2}-a_{3}=0$ are satisfied for $k_{1} \in(-7.30,26.53)$.

In Fig. 6 we have chosen $k_{1}=16.96$ and the initial conditions to be $\left(x_{0}, y_{0}, z_{0}\right)=(5,2,2)$. As can be seen from the figure, all states converge to their equilibria.

In order to get a faster response, one can easily consider the other two gains ( $k_{2}$ and $\left.k_{3}\right)$ in the control law. However, this introduces difficulties in the real implementation of the control system. We also note that in designing the stabilizing controllers, because we are utilizing the linearized version of the nonlinear system around its equilibrium, we should select the initial conditions near the corresponding equilibrium.

\section{Conclusions and future work}

Chaotic fractional order systems have an inherent potential in mechatronic applications, particularly in secure telecommunication systems where the main part of a transmitter-receiver configuration is the synchronization between master and slave blocks. In this article we analyzed the dynamical behavior of a novel fractional order chaotic system. The chaotic system generalizes the recent integer order system introduced in [46]. The local stability of the equilibria, using the fractional Routh-Hurwitz conditions, was studied. Furthermore, using Matignon's stability criteria, the system was shown to be chaotic in a wide parameter range, and to have an interesting complex dynamical behavior that varies
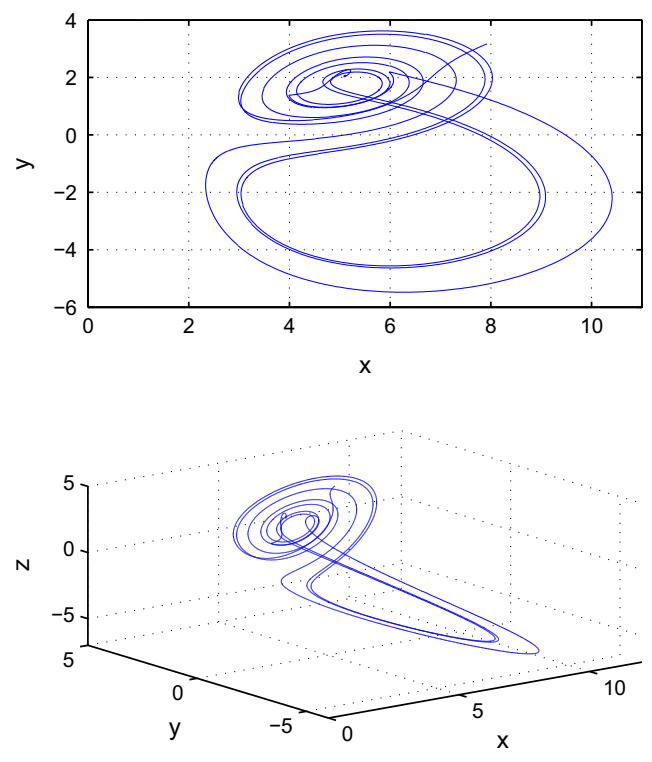

Fig. 6. Numerical results for the controlled system (13) with $a=3, b=2.7, c=4.7, d=2, h=9, k_{1}=16.96, k_{2}=k_{3}=0$, when the fractional order is $\alpha=0.90$ and initial conditions are $\left(x_{0}, y_{0}, z_{0}\right)=(5,2,2)$. 
according with the values of the parameters $a, b, c, d$, and $h$. The very rich nonlinear dynamics include chaos and period doubling bifurcations. Moreover, we derived a lower bound of the fractional order of differentiation for the system to remain chaotic. Analytical conditions for linear feedback control have been achieved. Our analysis is valid in a general format, in which all gains $k_{1}, k_{2}$, and $k_{3}$ are considered. However, and despite the complexity and the wide range of varieties, it has been shown that the system can be controlled by a single state-feedback controller. This possibility of stabilizing the system locally using only controller $k_{1}$ provides a simple and easy way to control the chaos, which can be crucial in a real implementation. Our state feedback approach can be applied to various chaotic fractional order systems. In particular, we claim that the techniques here developed can be used, without fundamental changes, in the synchronization of two fractional order chaotic systems in a secure telecommunication system. This is under study and will be addressed elsewhere.

Our state feedback approach for controlling the system is based on a Routh-Hurwitz analysis and cannot treat constraints on the actuating signal. To consider such saturation constraints on the control signal, a performance index should be defined. Minimizing the index subject to some constraints on the state and control signals cannot be considered analytically for the method here implemented. A direction for future research is to investigate how one can obtain a global stabilizing controller. To the best of our knowledge, the global stability of fractional systems is an interesting open question and available results reduce to those of $[62,63]$. For different stability concepts than the Lyapunov one adopted in our work, we refer the reader to [64].

\section{Acknowledgements}

This work was partially supported by FEDER through COMPETE, Operational Programme Factors of Competitiveness, and by Portuguese funds through the Center for Research and Development in Mathematics and Applications (CIDMA, University of Aveiro) and the Portuguese Foundation for Science and Technology (FCT), within project PEst-C/MAT/UI4106/2011 with COMPETE number FCOMP-01-0124-FEDER-022690. The authors are grateful to two anonymous referees for valuable comments and helpful suggestions.

\section{References}

[1] Miller KS, Ross B. An Introduction to the Fractional Calculus and Fractional Differential Equations. New York: A Wiley-Interscience Publication, Wiley; 1993.

[2] Hilfer R. Applications of Fractional Calculus in Physics. River Edge, NJ: World Sci. Publishing; 2000

[3] Debnath L. Recent applications of fractional calculus to science and engineering. Int. J. Math. Math. Sci. 2003;20(54):3413-42.

[4] Davis GB, Kohandel M, Sivaloganathan S, Tenti G. The constitutive properties of the brain paraenchyma: Part 2, Fractional derivative approach. Medi. Eng. Phys. 2006;28:455-9.

[5] Scalas E, Gorenflo R, Mainardi F. Fractional calculus and continuous-time finance. Physics A 2000;284(1-4):376-84

[6] Ahmad WM, El-Khazali R. Fractional-order dynamical models of love. Chaos Solitons Fractals 2007;33(4):1367-75

[7] Frederico GSF, Torres DFM. Fractional conservation laws in optimal control theory. Nonlinear Dynam. 2008;5393:215-22.

[8] Zhou P, Kuang F. A novel control method for integer orders chaos systems via fractional-order derivative. Discrete Dyn. Nat. Soc. 2011;2011:8. Art. ID 217843.

[9] Ross B. Fractional calculus and its applications. Lecture Notes in Mathematics, vol. 457. New York: Springer-Verlag; 1975.

[10] McBride A, Roach G. Fractional calculus. Research Notes in Mathematics, vol. 138. Boston: Pitman; 1985.

[11] Nishimoto K. Fractional calculus and its applications. Koriyama: Nihon University; 1990

[12] Mainardi F. Considerations on fractional calculus: interpretations and applications, in: Transform methods \& special functions, Varna '96, Bulgarian Acad. Sci., Sofia, 1998, pp. 594-597.
[13] Nigmatullin RR, Le Mehaute A. Is there a geometrical/physical meaning of the fractional integral with complex exponent? J. Non-Crystalline Solids 2005;351(33-36):2888-99.

[14] Podlubny I. Geometric and physical interpretation of fractional integration and fractional differentiation. Fract. Calc. Appl. Anal. 2002:5(4):367-86.

[15] Sabatier J, Merveillaut M, Malti R, Oustaloup A. How to impose physically coherent initial conditions to a fractional system? Commun. Nonlinear Sci. Numer. Simul. 2010;15(5):1318-26.

[16] Mozyrska D, Torres DFM. Minimal modified energy control for fractional linear control systems with the Caputo derivative. Carpathian J. Math. 2010;26(2):210-21.

[17] Mozyrska D, Torres DFM. Modified optimal energy and initial memory of fractional continuous-time linear systems. Signal Process 2011;91(3):379-85.

[18] Nigmatullin RR. A fractional integral and its physical interpretation. Theoret. Math. Phys. 1992;90(3):242-51.

[19] Rutman RS. On physical interpretations of fractional integration and differentiation. Theoret. Math. Phys. 1995;105(3):1509-19.

[20] Magin RL. Fractional calculus models of complex dynamics in biological tissues. Comput. Math. Appl. 2010;59(5):1586-93.

[21] West BJ. Fractal physiology and chaos in medicine. Studies of Nonlinear Phenomena in Life Science, vol. 1. Teaneck, NJ: World Sci. Publishing; 1990.

[22] Song L, Xu S, Yang J. Dynamical models of happiness with fractional order. Commun. Nonlinear Sci. Numer. Simul. 2010;15(3):616-28.

[23] Torvik PJ, Bagley RL. On the appearance of the fractional derivative in the behavior of real materials. Trans. ASME 1984;51:294-8.

[24] Chua LO, Itoh M, Kocarev L, Eckert K. Chaos synchronization in Chua's circuit. J. Circuits Systems Comput. 1993;3(1):93-108.

[25] Chen G, Dong X. From chaos to order. World Scientific Series on Nonlinear Science. Series A: Monographs and Treatises, vol. 24. River Edge, NJ: World Sci. Publishing; 1998.

[26] Kiani-B A, Fallahi K, Pariz N, Leung H. A chaotic secure communication scheme using fractional chaotic systems based on an extended fractional Kalman filter Commun. Nonlinear Sci. Numer. Simul. 2009;14(3):863-79.

[27] Nigmatullin RR, Omay T, Baleanu D. On fractional filtering versus conventional filtering in economics. Commun. Nonlinear Sci. Numer. Simul. 2010;15(4):979-86

[28] Wang T, Jia N. Chaos control and hybrid projective synchronization of several new chaotic systems. Appl. Math. Comput. 2012;218(13):7231-40.

[29] Couceiro MS, Ferreira NMF, Machado JAT. Application of fractional algorithms in the control of a robotic bird. Commun. Nonlinear Sci. Numer. Simul. 2010;15:895-910.

[30] Ladaci S, Loiseau JJ, Charef A. Fractional order adaptive high-gain controllers for a class of linear systems. Commun. Nonlinear Sci. Numer. Simul. 2008;13(4):707-14.

[31] Motallebzadeh F, Motlagh MRJ, Cherati ZR. Synchronization of different-order chaotic systems: adaptive active vs. optimal control. Commun. Nonlinear Sci. Numer. Simul. 2012;17(9):3643-57.

[32] Luo Y, Chen YQ, Ahn HS, Pi YG. Fractional order robust control for cogging effect compensation in PMSM position servo systems: stability analysis and experiments. Control Eng. Practice 2010;18:1022-36.

[33] Delavari H, Ghaderi R, Ranjbar A, Momani S. Fuzzy fractional order sliding mode controller for nonlinear systems. Commun. Nonlinear Sci. Numer. Simul. 2010;15(4):963-78

[34] Razminia A, Majd VJ, Baleanu D. Chaotic incommensurate fractional order Rössler system: active control and synchronization. Adv. Diff. Equ. 2011;2011(15):12.

[35] Magin RL. Fractional Calculus in Bioengineering. Connecticut: Begell House; 2006.

[36] Malinowska AB, Torres DFM. Introduction to the Fractional Calculus of Variations. London: Imp. Coll. Press; 2012.

[37] Oldham KB, Spanier J. The Fractional Calculus. New York: Academic Press; 1974.

[38] Oustaloup A. La derivation non entiere: Theorie. synthese et applications. Paris: Hermes; 1995.

[39] Podlubny I. Fractional differential equations. Mathematics in Science and Engineering, Vol. 198. San Diego, CA: Academic Press; 1999.

[40] Cafagna D. Fractional calculus: a mathematical tool from the past for the present engineer. IEEE Trans. Indust. Electronic 2007;1(2):35-40.

[41] Li C, Deng W. Remarks on fractional derivatives. Appl. Math. Comput 2007; 187(2):777-84.

[42] Wang Y, Li C. Does the fractional Brusselator with efficient dimension less than 1 have a limit cycle? Phys. Lett. A 2007;363(5-6):414-9.

[43] Matignon D. Stability results for fractional differential equations with applications to control processing, in: Computational Engineering in Systems and Applications, Multi-conference, vol. 2, IMACS, IEEE-SMC Proceedings, Lille, France, pp. 963-968, July 1996.

[44] Deng W, Li C, Lü J. Stability analysis of linear fractional differential system with multiple time delays. Nonlinear Dynam. 2007;48(4):409-16.

[45] Matouk AE. Chaos, feedback control and synchronization of a fractional-order modified autonomous Van der Pol-Duffing circuit. Commun. Nonlinear Sci. Numer. Simul. 2011;16(2):975-86.

[46] Dadras S, Momeni HR. A novel three-dimensional autonomous chaotic system generating two, three and four-scroll attractors. Phys. Lett. A 2009;373940:3637-42.

[47] Barbara C, Silvano C. Hyperchaotic behaviour of two bi-directionally Chua's circuits. Int. J. Circ. Theory Appl. 2002;30(6):625-37. 
[48] Grassi G, Mascolo S. A system theory approach for designing cryptosystems based on hyperchaos. IEEE Trans. Circuits Syst. I 1999;46(9):1135-8.

[49] Udaltsov VS, Goedgebuer JP, Larger L, Cuenot JB, Levy P, Rhodes WT. Communicating with hyperchaos: the dynamics of a DNLF emitter recovery of transmitted information. Opt. Spectrosc. 2003;95(1):114-8.

[50] Vicente R, Daudén J, Colet P, Toral R. Analysis and characterization of the hyperchaos generated by a semiconductor laser subject to a delayed feedback loop. IEEE J. Quant. Electron. 2005;41(4):541-8.

[51] Cenys A, Tamaservicius A, Baziliauskas A, Krivickas R, Lindberg E. Hyperchaos in coupled Colpitts oscillators. Chaos Solitons Fract. 2003;17(2):349-53.

[52] Silva CP. Shil' nikov's theorem-a tutorial. IEEE Trans. Circuits Systems I Fund. Theory Appl. 1993;40(10):675-82.

[53] Chu F, Zhang Z. Bifurcation and chaos in rub-impact Jeffcott rotor system. J. Sound Vib. 1998;210(1):1-18.

[54] Chu F, Lu W. Experimental observation of nonlinear vibrations in a rub-impact rotor system. J. Sound Vib. 2005;283:621-43.

[55] Ehrich FF. Some observations of chaotic vibration phenomena in high-speed rotor dynamics. ASME J. Vib. Acoust. 1991;113:50-7.

[56] Goldman P, Muszynska A. Chaotic behavior of rotor/stator systems with rubs. Trans. ASME J. Eng. Gas Turbine Power 1994;116:692-701.
[57] Lin F, Schoen MP, Korde UA. Numerical investigation with rub-related vibration in rotating machinery. J. Vib. Control 2001;7(6):833-48.

[58] Houfek L, Houfek M, Krejsa J, Kratochvil C, Kolacny J, Nykodym P. Bifurcation and chaos in electromechanical drive systems with small MPTPRS. Engineering Mechanics 2008;15(5):329-36.

[59] Tavazoei MS, Haeri M. Chaotic attractors in incommensurate fractional order systems. Physics D 2008;237(20):2628-37.

[60] Diethelm K, Ford NJ. Analysis of fractional differential equations. J. Math. Anal. Appl. 2002;265(2):229-48.

[61] Diethelm K, Ford NJ, Freed AD. A predictor-corrector approach for the numerical solution of fractional differential equations. Nonlinear Dynam. 2002;29(1-4):3-22.

[62] Li Y, Chen Y, Podlubny I. Mittag-Leffler stability of fractional order nonlinear dynamic systems. Automatica J. IFAC 2009;45(8):1965-9.

[63] Li Y, Chen Y, Podlubny I. Stability of fractional-order nonlinear dynamic systems: Lyapunov direct method and generalized Mittag-Leffler stability. Comput. Math. Appl. 2010;59(5):1810-21.

[64] Wang J, Lv L, Zhou Y. New concepts and results in stability of fractional differential equations. Commun. Nonlinear Sci. Numer. Simul. 2012;17(6):2530-8. 